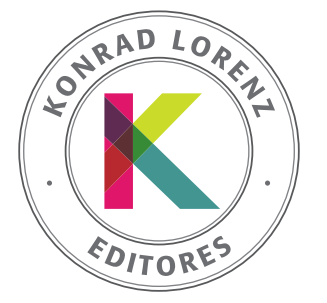

\title{
Realidad socioeconómica de personas en condición de desplazamiento forzado en Colombia: revisión sistemática
}

\author{
Myriam Sierra-Puentes y Andrea Correa-Chica
}

Fundación Universitaria Konrad Lorenz, Bogotá, Colombia

Recibido el 15 de septiembre de 2018; aceptado el 1 de marzo de 2019

\author{
PALABRAS CLAVE \\ migración forzada, \\ desplazamiento \\ forzado interno, \\ seguridad económica, \\ comportamiento \\ del consumidor
}

\section{KEYWORDS}

Forced migration, internal forced displacement, economic security, consumer behavior

\begin{abstract}
Resumen Colombia es el país con mayor número de población en condición de desplazamiento forzado interno (CDFI) en el mundo. Una de las necesidades más apremiantes que enfrentan las personas afectadas es reestructurar su área económica. Puesto que se dificulta la consecución de fuentes permanentes de ingreso. Por tanto, realizamos una revisión sistemática de la literatura para conocer las prácticas de consumo y fuentes de ingreso de las personas en CDFI en Colombia y qué metodologías se emplean para abordar el estudio de esta realidad. La búsqueda se ejecutó en las bases de datos Redalyc y Scopus de artículos originales publicados a partir de 2006. Se encontraron 6.146. De estos, 26 fueron elegibles y proporcionaron información sobre: la seguridad alimentaria (42.3\%), el estado de salud y/o acceso a sanidad (26.9\%); las condiciones de vivienda (45.5\%), el acceso a servicios públicos (27.3\%); la tenencia de propiedades y/o bienes (26.9\%); la situación laboral (76.9\%), el nivel de escolaridad y acceso a la educación (46.2\%) y el acceso a ayuda humanitaria (15.4\%) de personas en CDFI en Colombia. Estos resultados permiten evidenciar la vulnerabilidad socioeconómica a la que se enfrentan las personas en CDFI en Colombia.

(c) 2019 Fundación Universitaria Konrad Lorenz. Este es un artículo Open Access bajo la licencia CC BY-NC-ND (http://creativecommons.org/licenses/bync-nd/4.0/).
\end{abstract}

Socio-economic reality of people under forced displacement conditions in Colombia: A systematic review

Colombia has the largest number of internally displaced persons (IDPs) in the world. One of the most pressing needs facing affected people is to restructure their economic base, as it is difficult to obtain permanent sources of income. As such, we conducted a systematic review of the literature to learn about IDPs' consumption practices and sources of income in Colombia and what methodologies are used to address the study of this reality. We searched the Redalyc and Scopus databases of original articles published from 2006. Of these, 26 were eligible and provided information on: food security $(42.3 \%)$, health

* Autor para correspondencia.

Correo electrónico: myriamc.sierrap@konradlorenz.edu.co

http://dx.doi.org/10.14349/sumapsi.2019.v26.n2.1

0120-0534/@ 2019 Fundación Universitaria Konrad Lorenz. Este es un artículo Open Access bajo la licencia CC BY-NC-ND (http://creativecommons.org/licenses/by-nc-nd/4.0/). 
status and/or access to health (26.9\%); housing conditions (45.5\%), access to public services (27.3\%); tenure of property and/or goods (26.9\%); employment situation $(76.9 \%)$, level of schooling and access to education (46.2\%) and IDPs' access to humanitarian aid (15.4\%) in Colombia. These results highlight the socioeconomic vulnerability faced by IDPs in Colombia.

(c) 2019 Fundación Universitaria Konrad Lorenz. This is an open access article under the CC BYNC-ND license (http://creativecommons.org/licenses/bync-nd/4.0/).

Colombia ha vivido más de 60 años en conflicto armado. La prolongación de la violencia generó que la población civil fuera víctima de ataques directos, reclutamiento forzado, amenazas, desapariciones, muertes y ejecuciones selectivas, desplazamiento forzado interno (DFI), entre otros (Carrillo, 2010; Centro Nacional de Memoria Histórica, 2013). La violencia se ha ejercido con el fin de impedir la acción colectiva, mantener control sobre la tierra, fortalecer bastiones territoriales y debilitar el apoyo del oponente (Bozzoli, Bruck \& Wald, 2012; Ibáñez \& Vélez, 2008).

Dentro de los principales factores causales de esta confrontación está la lucha por el control de territorios estratégicos para los diferentes actores armados. Por ello, el mayor impacto del conflicto armado se presenta en zonas rurales, en áreas ricas en recursos (Aysa-Lastra, 2011). La población rural con frecuencia es objeto de amenaza por parte de los actores armados que tienen como propósito emplear las tierras de los civiles para su beneficio particular; y como resultado de esto, despojan a la población de su hábitat generando altas tasas de DFI de campesinos o pequeños productores (Bozzoli et al., 2012).

Por tanto, el DFI es la reacción por parte de la población civil a una amenaza o ataque que generan los actores armados y que pone en peligro su vida y/o la de su familia (Ibáñez \& Vélez, 2008). Desde el marco jurídico colombiano una persona se define como desplazada cuando migra de manera forzada dentro del territorio nacional y abandona su localidad de residencia y/o actividades económicas habituales debido a que su vida, su integridad física, su seguridad o libertad personal han sido vulneradas o se encuentran amenazadas como resultado del conflicto armado interno, disturbios y tensiones interiores, violencia generalizada, violaciones masivas de derechos humanos, infracciones al derecho internacional humanitario $u$ otras circunstancias emanadas de las situaciones anteriores que puedan alterar o alteren drásticamente el orden público (Congreso de la República de Colombia, 1997).

El DFI es el hecho que más víctimas ha cobrado a causa de la violencia en Colombia (Mogollón Pérez \& Vázquez Navarrete, 2006; Prada, Herrán \& Villamizar, 2010). Entre las cifras de DFI reportadas por organizaciones nacionales e internacionales se encuentran diferencias importantes. Sin embargo, todos estos organismos coinciden en ubicar a Colombia como uno de los países con el mayor número de población en CDFI en el mundo. Lo que representa una de las crisis humanitarias más relevantes de los últimos tiempos y se convierte en uno de los mayores desafíos para el Estado colombiano (Bohada, 2010; Muggah, 2000).

La población en CDFI migra con frecuencia de zonas rurales a urbanas, principalmente, a ciudades como Medellín, Cali o Bogotá (ciudad capital) y sus alrededores. Este cambio genera grandes transformaciones a nivel individual, social y económico (Carrillo, 2010; Hayes-Conroy \& Sweet, 2014).

En primer lugar, a nivel individual el DFI ocasiona: alteraciones en el bienestar emocional y la salud mental, disminución de la salud física, decremento de la calidad de vida e interrupción en la formación educativa (Aysa-Lastra, 2011; Carrillo, 2010; Machado-Duque \& Calderón-Flórez, 2014; Macuacé, 2009; Mogollón Pérez \& Vázquez Navarrete, 2006).

En segunda instancia, a nivel social el DFI enfrenta a las personas a condiciones de hacinamiento, alteración en la estructura y el tamaño de los hogares, desintegración familiar, aumento de la violencia intrafamiliar y con la pareja; dificultades en la crianza, pérdida de redes sociales, estigmatización y exclusión social; e incluso, cambio en los roles de género, puesto que en los lugares de asentamiento las mujeres tienden a asumir papeles como proveedoras y protectoras del hogar (Aysa-Lastra, 2011; Carrillo, 2010; Guevara Fletcher, 2011; Hayes-Conroy \& Sweet, 2014; Hérnandez Bello \& Gutiérrez, 2008; Machado-Duque \& Calderón-Flórez, 2014; Mogollón Pérez \& Vázquez Navarrete, 2006).

Así mismo, el DFI ocasiona un deterioro importante en los ingresos económicos de las familias, dado que enfrenta a las personas cabezas de hogar al desempleo o a empleos precarios de carácter informal. También produce constante incertidumbre ante la dificultad de obtener fuentes alternativas de ingreso; limitaciones para cubrir las necesidades básicas, como la alimentación; pérdida de activos; inconvenientes para acceder a los servicios de salud y servicios públicos; dependencia económica; condiciones de riesgo en vivienda y distorsiones en la economía local, regional y nacional (Aysa-Lastra, 2011; Carranza Romero, Dueñas Herrera \& González Espitia, 2011; Carrillo, 2010; HayesConroy \& Sweet, 2014; Hérnandez Bello \& Gutiérrez, 2008; Machado-Duque \& Calderón-Flórez, 2014; Mogollón-Pérez \& Vázquez, 2008).

A pesar de los impactos socioeconómicos que genera el DFI en las personas víctimas de este flagelo los estudios que se centran en investigar estas particularidades son limitados. Así mismo, no se cuenta con un recurso de síntesis sobre los hallazgos empíricos al respecto. Por tanto, existe la necesidad de analizar la evidencia científica con el fin de: (a) tener mayor claridad sobre los efectos socioeconómicos que genera el DFI en las personas que se vieron obligadas a migrar; y (b) proporcionar una síntesis sobre las metodologías empleadas para abordar este fenómeno de estudio (Petticrew \& Roberts, 2008).

Para responder a esta necesidad, realizamos una revisión sistemática de la literatura científica con el objetivo de identificar las prácticas de consumo y fuentes de ingreso de las personas en CDFI en Colombia y qué metodologías se emplean para abordar el estudio de esta realidad. 


\section{Método}

\section{Estrategia de búsqueda}

Para obtener los estudios se utilizaron como estrategia de búsqueda las combinaciones desplazamiento forzado $\mathrm{Y}$ Colombia, forced displacement AND Colombia, forced displacement Colombia AND economy, forced migration AND Colombia y migración forzada Y Colombia. Además, los artículos indexados con las palabras clave "desplazamiento forzado", "desplazamiento" y "forced displacement" se identificaron utilizando el método de palabras clave individuales. Por último, referencias citadas dentro de los artículos obtenidos también fueron revisadas.

La figura 1 ilustra el proceso de selección de los estudios incluidos en esta revisión sistemática. En la búsqueda inicial se identificaron 6.146 artículos. Después de revisar los títulos y resúmenes, se descartaron 6.084, esto debido a que los artículos estudiaban temáticas del DFI que no profundizaban en aspectos socioeconómicos o las muestras estudiadas eran de otros países. Se preseleccionaron 62 artículos de acuerdo con el contenido del resumen para lectura a texto completo, de estos se eligieron 29 y finalmente de acuerdo con los criterios de inclusión 26 fueron escogidos para la extracción de datos. Todo el proceso se realizó siguiendo las directrices de la declaración Prisma (Urrutia \& Bonfill, 2010).

Se optó por aquellos artículos en los que: (1) los participantes de los estudios fueron personas que se vieron forzadas a migrar dentro de territorio colombiano; (2) se centraron en estudiar las fuentes de obtención de recursos económicos y los procesos socioculturales que realizan las personas en CDFI para la apropiación y uso de productos y/o servicios. Se excluyeron artículos que se centraban en realizar análisis únicamente sobre el impacto socioeconómico del DFI en la población receptora o en los que las prácticas de consumo y fuentes de ingreso no fueran las temáticas centrales de investigación.

La revisión sistemática de la literatura científica recolectó estudios producidos desde 2006. La búsqueda de información se efectuó entre julio y septiembre de 2017. Se incluyeron estudios empíricos con metodología cuantitativa y cualitativa de acuerdo con la clasificación de Montero y León (2007). Los artículos se obtuvieron a través de las bases de datos Scopus (Elsevier) y Redalyc (Red de Revistas Científicas de América Latina y el Caribe, España y Portugal).

\section{Selección y extracción de datos}

Se extrajeron las siguientes características de cada estudio: año de publicación, idioma, lugar de publicación, metodología, tipo de estudio, diseño, tipo de muestra, género, entidad realizadora, fuente de financiación, cobertura de la investigación y las temáticas centrales de estudio. Además, se recolectaron los textos de los apartados de: título, resumen, palabras clave, resultados y discusión de todos los artículos incluidos.

\section{Resultados}

Los resultados que se presentan a continuación corresponden a un análisis descriptivo y estadístico de datos textuales que contempla variables de tipo institucional, geográfico y metodológico de los 26 artículos originales (tabla 1) que investigaron las prácticas de consumo y fuentes de ingreso de las personas en CDFI en Colombia.

\section{Análisis descriptivo de los estudios}

Los estudios se publicaron en revisitas de América del Sur (50\%), en países como Colombia (42.3\%), seguido de Brasil (3.8\%) y Venezuela (3.8\%); en Europa (26.9\%), en países como Reino Unido (11.5\%), Países Bajos (7.7\%) y España (7.7\%); y, por último, algunos en América del Norte (15.4\%) y América Central (7.7\%).

A nivel metodológico los estudios emplearon, principalmente, enfoques cuantitativos (73.1\%). Pocos estudios utilizaron un enfoque cualitativo (19.2\%) o mixto (7.7\%). En cuanto al tipo de estudio, se contó con investigaciones de alcance descriptivo (42.3\%), ex post facto (30.8\%) y de panel (7.7\%) y/o evolución de grupo (3.8\%) con perspectiva cuantitativa y/o mixta. Desde la metodología cualitativa solo se

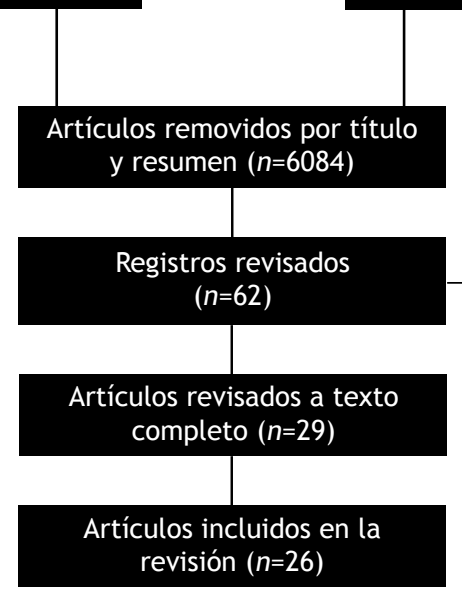


reportaron investigaciones de tipo fenomenológico. Esto se puede deber al objetivo mismo de este tipo de método que es describir la experiencia, en este caso del DFI, sin recurrir a explicaciones causales (Moreno, 2005). En cuanto a los estudios cuantitativos el diseño más empleado fue el trans- versal (61.5\%), fueron pocos los estudios que recurrieron a un diseño longitudinal (19.2\%).

Respecto a las muestras se identificó que los estudios contaron con participación de ambos géneros (84.6\%) a excepción de los que investigaron solo a personas de género

Tabla 1.

\begin{tabular}{|c|c|c|c|c|c|c|c|}
\hline Autor & Metodología & $\begin{array}{l}\text { Tipo de } \\
\text { estudio }\end{array}$ & Diseño & Tema estudiado & Muestra & Género & Alcance \\
\hline $\begin{array}{l}\text { Arias, lbáñez \& } \\
\text { Querubín (2014) }\end{array}$ & Cuantitativa & Descriptivo & Transversal & $\begin{array}{c}\text { Tenencia de propiedades } \\
\text { y/o bienes }\end{array}$ & $\begin{array}{l}\text { Unidades } \\
\text { familiares }\end{array}$ & $\begin{array}{l}\text { Femenino y } \\
\text { masculino }\end{array}$ & Nacional \\
\hline $\begin{array}{l}\text { Aysa-Lastra } \\
\text { (2011) }\end{array}$ & Cuantitativa & $\begin{array}{l}\text { Ex post } \\
\text { facto }\end{array}$ & Transversal & $\begin{array}{l}\text { Seguridad alimentaria, Condi- } \\
\text { ciones de vivienda, Acceso a } \\
\text { servicios públicos, Tenencia de } \\
\text { propiedades y/o bienes, Situa- } \\
\text { ción laboral, Nivel de escolari- } \\
\text { dad y acceso a la educación }\end{array}$ & Adulta & $\begin{array}{l}\text { Femenino y } \\
\text { masculino }\end{array}$ & Local \\
\hline
\end{tabular}

\begin{tabular}{|c|c|c|c|c|c|c|c|}
\hline $\begin{array}{l}\text { Bozzoli et al. } \\
\text { (2012) }\end{array}$ & Cuantitativa & Panel & Longitudinal & $\begin{array}{l}\text { Seguridad alimentaria, Esta- } \\
\text { do de salud y/o acceso a sa- } \\
\text { nidad, Condiciones de vivien- } \\
\text { da, Tenencia de propiedades } \\
\text { y/o bienes, Situación laboral, } \\
\text { Nivel de escolaridad y acceso } \\
\text { a la educación }\end{array}$ & Adulta & $\begin{array}{l}\text { Femenino y } \\
\text { masculino }\end{array}$ & Nacional \\
\hline Carrillo (2010) & Cuantitativa & Descriptivo & Transversal & $\begin{array}{l}\text { Nivel de escolaridad y acce- } \\
\text { so a la educación, Situación } \\
\text { laboral, Seguridad alimenta- } \\
\text { ria, Condiciones de vivienda }\end{array}$ & $\begin{array}{l}\text { Datos } \\
\text { oficiales }\end{array}$ & $\begin{array}{l}\text { Femenino y } \\
\text { masculino }\end{array}$ & Nacional \\
\hline $\begin{array}{l}\text { Castiblanco } \\
\text { Moreno (2016) }\end{array}$ & Cualitativa & $\begin{array}{l}\text { Fenomeno- } \\
\text { lógico }\end{array}$ & N. A. & Situación laboral & Adulta & Femenino & Nacional \\
\hline $\begin{array}{l}\text { Dueñas, } \\
\text { Palacios \& } \\
\text { Zuluaga (2014) }\end{array}$ & Cuantitativa & Panel & Longitudinal & $\begin{array}{c}\text { Situación laboral } \\
\text { Condiciones de vivienda }\end{array}$ & $\begin{array}{l}\text { Datos } \\
\text { oficiales }\end{array}$ & $\begin{array}{l}\text { Femenino y } \\
\text { masculino }\end{array}$ & Nacional \\
\hline $\begin{array}{l}\text { Espinosa } \\
\text { Menéndez, } \\
\text { Galindo Rosero, } \\
\text { Bastidas Lopera } \\
\text { \& Monsalve } \\
\text { Rojas (2012) }\end{array}$ & Cuantitativa & Descriptivo & Transversal & $\begin{array}{l}\text { Nivel de escolaridad y acceso } \\
\text { a la educación, Situación la- } \\
\text { boral, Tenencia de propieda- } \\
\text { des y/o bienes, Condiciones } \\
\text { de vivienda, Acceso a ayuda } \\
\text { humanitaria }\end{array}$ & $\begin{array}{l}\text { Datos } \\
\text { oficiales }\end{array}$ & $\begin{array}{l}\text { Femenino y } \\
\text { masculino }\end{array}$ & Departamental \\
\hline $\begin{array}{l}\text { Gamboa- } \\
\text { Delgado, } \\
\text { López-Barbosa, } \\
\text { Vera-Cala \& } \\
\text { Prada-Gómez } \\
\text { (2007) }\end{array}$ & Cuantitativa & Descriptivo & Transversal & Seguridad alimentaria & Infantil & $\begin{array}{l}\text { Femenino y } \\
\text { masculino }\end{array}$ & Local \\
\hline $\begin{array}{l}\text { Guevara Flet- } \\
\text { cher (2011) }\end{array}$ & $\begin{array}{l}\text { Cualitativa- } \\
\text { Cuantitativa }\end{array}$ & $\begin{array}{l}\text { Evolución } \\
\text { de grupo }\end{array}$ & Longitudinal & $\begin{array}{l}\text { Seguridad alimentaria, Estado } \\
\text { de salud y/o acceso a sanidad, } \\
\text { Condiciones de vivienda, Acce- } \\
\text { so a servicios públicos, Situa- } \\
\text { ción laboral, Nivel de escola- } \\
\text { ridad y acceso a la educación, } \\
\text { Acceso a ayuda humanitaria }\end{array}$ & $\begin{array}{l}\text { Unidades } \\
\text { familiares }\end{array}$ & $\begin{array}{l}\text { Femenino y } \\
\text { masculino }\end{array}$ & Local \\
\hline $\begin{array}{l}\text { Hayes-Conroy } \\
\text { \& Sweet (2014) }\end{array}$ & Cualitativa & $\begin{array}{l}\text { Fenomeno- } \\
\text { lógico }\end{array}$ & N.A & Seguridad alimentaria & Adulta & Femenino & Local \\
\hline
\end{tabular}


Tabla 1.

\begin{tabular}{|c|c|c|c|c|c|c|c|}
\hline Autor & Metodología & $\begin{array}{l}\text { Tipo de } \\
\text { estudio }\end{array}$ & Diseño & Tema estudiado & Muestra & Género & Alcance \\
\hline $\begin{array}{l}\text { Hérnandez } \\
\text { Bello \& } \\
\text { Gutiérrez } \\
(2008)\end{array}$ & $\begin{array}{l}\text { Cualitativa - } \\
\text { Cuantitativa }\end{array}$ & Descriptivo & Transversal & $\begin{array}{l}\text { Seguridad alimentaria, Estado } \\
\text { de salud y/o acceso a sanidad, } \\
\text { Condiciones de vivienda, Si- } \\
\text { tuación laboral, Nivel de esco- } \\
\text { laridad y acceso a la educación }\end{array}$ & $\begin{array}{l}\text { Unidades } \\
\text { familiares }\end{array}$ & $\begin{array}{l}\text { Femenino y } \\
\text { masculino }\end{array}$ & Local \\
\hline $\begin{array}{l}\text { Herrán \& Patiño } \\
\text { (2015) }\end{array}$ & Cuantitativa & Descriptivo & Longitudinal & $\begin{array}{l}\text { Seguridad alimentaria, Condi- } \\
\text { ciones de vivienda, Acceso a } \\
\text { servicios públicos, Situación } \\
\text { laboral, Nivel de escolaridad y } \\
\text { acceso a la educación }\end{array}$ & Adulta & $\begin{array}{l}\text { Femenino y } \\
\text { masculino }\end{array}$ & Local \\
\hline $\begin{array}{l}\text { Ibáñez \& Moya } \\
(2010)\end{array}$ & Cuantitativa & $\begin{array}{l}\text { Ex post } \\
\text { facto }\end{array}$ & Transversal & $\begin{array}{l}\text { Seguridad alimentaria, Condi- } \\
\text { ciones de vivienda, Acceso a } \\
\text { servicios públicos, Tenencia de } \\
\text { propiedades y/o bienes, Situa- } \\
\text { ción laboral, Nivel de escolari- } \\
\text { dad y acceso a la educación, } \\
\text { Acceso a ayuda humanitaria }\end{array}$ & $\begin{array}{l}\text { Unidades } \\
\text { familiares }\end{array}$ & $\begin{array}{l}\text { Femenino y } \\
\text { masculino }\end{array}$ & Nacional \\
\hline $\begin{array}{l}\text { Ibáñez \& Vélez } \\
(2008)\end{array}$ & Cuantitativa & $\begin{array}{l}\text { Ex post } \\
\text { facto }\end{array}$ & Transversal & $\begin{array}{l}\text { Acceso a servicios públicos- } \\
\text { Tenencia de propiedades y/o } \\
\text { bienes Situación laboral Nivel } \\
\text { de escolaridad y acceso a la } \\
\text { educación }\end{array}$ & $\begin{array}{l}\text { Unidades } \\
\text { familiares }\end{array}$ & $\begin{array}{l}\text { Femenino y } \\
\text { masculino }\end{array}$ & Local \\
\hline $\begin{array}{l}\text { Machado-Duque } \\
\text { \& Calderón- } \\
\text { Flórez (2014) }\end{array}$ & Cuantitativa & $\begin{array}{l}\text { Ex post } \\
\text { facto }\end{array}$ & Transversal & $\begin{array}{l}\text { Seguridad alimentaria, Situa- } \\
\text { ción laboral, Nivel de escola- } \\
\text { ridad y acceso a la educación }\end{array}$ & Infantil & $\begin{array}{l}\text { Femenino y } \\
\text { masculino }\end{array}$ & Local \\
\hline Macuacé (2009) & Cuantitativa & Descriptivo & Transversal & $\begin{array}{l}\text { Condiciones de vivienda, Estado } \\
\text { de salud y/o acceso a sanidad, } \\
\text { Acceso a servicios públicos, } \\
\text { Tenencia de propiedades y/o } \\
\text { bienes, Situación laboral Nivel } \\
\text { de escolaridad y acceso a la } \\
\text { educación }\end{array}$ & Adulta & $\begin{array}{l}\text { Femenino y } \\
\text { masculino }\end{array}$ & Local \\
\hline $\begin{array}{l}\text { Mogollón Pérez } \\
\text { \& Vázquez } \\
\text { Navarrete } \\
(2006)\end{array}$ & Cualitativa & $\begin{array}{l}\text { Fenomeno- } \\
\text { lógico }\end{array}$ & N.A & $\begin{array}{l}\text { Estado de salud } \\
\text { y/o acceso a sanidad }\end{array}$ & Adulta & Femenino & Local \\
\hline $\begin{array}{l}\text { Mogollón- } \\
\text { Pérez \& } \\
\text { Vázquez (2008) }\end{array}$ & Cualitativa & $\begin{array}{l}\text { Fenomeno- } \\
\text { lógico }\end{array}$ & N.A & $\begin{array}{l}\text { Estado de salud } \\
\text { y/o acceso a sanidad }\end{array}$ & Adulta & $\begin{array}{l}\text { Femenino y } \\
\text { masculino }\end{array}$ & Local \\
\hline $\begin{array}{l}\text { Pareja Amador } \\
\text { \& Domínguez } \\
(2014)\end{array}$ & Cualitativa & Descriptivo & N.A & Situación laboral & Adulta & Femenino & Nacional \\
\hline $\begin{array}{l}\text { Patiño \& } \\
\text { Herrán (2013) }\end{array}$ & Cuantitativa & Descriptivo & Transversal & Situación laboral & Adulta & $\begin{array}{c}\text { Femenino y } \\
\text { masculino }\end{array}$ & Local \\
\hline $\begin{array}{l}\text { Prada Gómez, } \\
\text { Herrán Falla \& } \\
\text { Ortiz Cárdenas } \\
(2008)\end{array}$ & Cuantitativa & Descriptivo & Transversal & Seguridad alimentaria & $\begin{array}{l}\text { Unidades } \\
\text { familiares }\end{array}$ & $\begin{array}{l}\text { Femenino y } \\
\text { masculino }\end{array}$ & Local \\
\hline $\begin{array}{l}\text { Prada et al. } \\
\text { (2010) }\end{array}$ & Cuantitativa & Descriptivo & Transversal & $\begin{array}{l}\text { Seguridad alimentaria } \\
\text { Situación laboral }\end{array}$ & $\begin{array}{l}\text { Unidades } \\
\text { familiares }\end{array}$ & $\begin{array}{c}\text { Femenino y } \\
\text { masculino }\end{array}$ & Local \\
\hline $\begin{array}{l}\text { Sánchez Isaza, } \\
\text { Cataño Ordóñez } \\
\text { \& Ordóñez } \\
\text { (2016) }\end{array}$ & Cuantitativa & $\begin{array}{l}\text { Ex post } \\
\text { facto }\end{array}$ & Transversal & $\begin{array}{l}\text { Estado de salud y/o acceso } \\
\text { a sanidad Situación laboral }\end{array}$ & $\begin{array}{l}\text { Unidades } \\
\text { familiares }\end{array}$ & $\begin{array}{l}\text { Femenino y } \\
\text { masculino }\end{array}$ & Nacional \\
\hline
\end{tabular}


Tabla 1.

\begin{tabular}{|c|c|c|c|c|c|c|c|}
\hline Autor & Metodología & $\begin{array}{l}\text { Tipo de } \\
\text { estudio }\end{array}$ & Diseño & Tema estudiado & Muestra & Género & Alcance \\
\hline $\begin{array}{l}\text { Silva Arias \& } \\
\text { Guataquí Roa } \\
\text { (2008) }\end{array}$ & Cuantitativa & $\begin{array}{l}\text { Ex post } \\
\text { facto }\end{array}$ & Longitudinal & $\begin{array}{l}\text { Situación laboral, Nivel de } \\
\text { escolaridad y acceso a la } \\
\text { educación, Acceso a ayuda } \\
\text { humanitaria }\end{array}$ & $\begin{array}{l}\text { Datos } \\
\text { oficiales }\end{array}$ & $\begin{array}{l}\text { Femenino y } \\
\text { masculino }\end{array}$ & Nacional \\
\hline $\begin{array}{l}\text { Silva Arias \& } \\
\text { Sarmiento Espi- } \\
\text { nel (2013) }\end{array}$ & Cuantitativa & $\begin{array}{l}\text { Ex post } \\
\text { facto }\end{array}$ & Transversal & Situación laboral & $\begin{array}{l}\text { Datos } \\
\text { oficiales }\end{array}$ & $\begin{array}{l}\text { Femenino y } \\
\text { masculino }\end{array}$ & Nacional \\
\hline $\begin{array}{l}\text { Silva \& Guata- } \\
\text { quí (2011) }\end{array}$ & Cuantitativa & $\begin{array}{l}\text { Ex post } \\
\text { facto }\end{array}$ & Transversal & Situación laboral & $\begin{array}{c}\text { Datos } \\
\text { oficiales }\end{array}$ & $\begin{array}{l}\text { Femenino y } \\
\text { masculino }\end{array}$ & Nacional \\
\hline
\end{tabular}

femenino (15.4\%). Así mismo, las unidades de análisis de los estudios fueron: individuos con mayoría de edad (38.5\%), familias (hogares, $30.8 \%$ ) y en otros casos emplearon datos oficiales del gobierno $u$ otras entidades (23.1\%) referente a encuestas nacionales, censos o bases de datos institucionales. Fueron pocos los estudios que contaron con participación únicamente de individuos en edad infantil (7.7\%).

Con base en la filiación institucional, se identificó que las investigaciones fueron realizadas por instituciones universitarias $(88.4 \%)$, seguido de organizaciones no gubernamentales (ONG, 7.7\%) y entidades de gobierno (3.8\%). Las fuentes de financiación reportadas fueron: las universidades $(65.3 \%)$, las alianzas público-privadas (15.4\%), seguido de las ONG (7.7\%), entidades de Estado (7.7\%) o alianzas entre entidades estatales (3.8\%). La cobertura de las investigaciones fue local $(53.8 \%)$ y nacional $(42.3 \%)$ principalmente, y en menor medida, con alcance departamental (3.8\%).

\section{Temáticas de estudio}

Respecto a las prácticas de consumo y fuentes de ingreso de las personas en CDFI en Colombia los temas que los estudios investigaron fueron: la seguridad alimentaria, el estado de salud $y / o$ acceso a sanidad, las condiciones de vivienda, el acceso a servicios públicos, la tenencia de propiedades y/o bienes, la situación laboral, el nivel de escolaridad y el acceso a la educación y el acceso a ayuda humanitaria.

La seguridad alimentaria fue investigada por el $42.3 \%$ de los estudios. Se reportó que la calidad de la dieta era deficiente. Los factores que se relacionaron con la deficiencia alimentaria fueron: (a) la frecuencia y lugar de compra de los alimentos así como la dependencia económica, debido a que después del DFI la compra tendía a ser diaria y se recurría con frecuencia al crédito para acceder al alimento; (b) la prevalencia de jefatura femenina en los hogares, puesto que las mujeres tendían a tener ingresos inferiores en comparación con los hombres; (c) los impedimentos para producir alimentos, dado que la mayoría de la personas en CDFI provenía de zonas rurales y producían gran parte de su dieta, pero después casi el total de los alimentos tenía que ser comprado.

Es evidente, por tanto, que una de las necesidades más apremiantes de las personas en CDFI es la alimentación y resulta ser un indicador de las condiciones de vida de estas unidades familiares. Esto pone de manifiesto la falta de cumplimiento del derecho a una alimentación equilibrada, factor que repercute en el deterioro del bienestar general y, en especial, de los infantes (Gamboa-Delgado et al., 2007; Guevara Fletcher, 2011; Hayes-Conroy \& Sweet, 2014; Hérnandez Bello \& Gutiérrez, 2008; Herrán \& Patiño, 2015; Machado-Duque \& Calderón-Flórez, 2014; Prada et al., 2010; Prada Gómez et al., 2008).

El estado de salud y/o el acceso a la sanidad lo investigaron el $26.9 \%$ de los estudios. Estos reportaron una alta presencia de cuadros de desnutrición, alta probabilidad de adquisición de enfermedades infecciosas debido a las condiciones de vivienda, problemas gastrointestinales y enfermedades crónicas e incapacitantes en las personas en CDFI.

En cuanto al estado psicológico se reportaron algunos diagnósticos tales como: alteraciones en el bienestar emocional, ansiedad, intento de suicidio, depresión, además, problemas de comportamiento en población infantil y adolescente. También se reportó, en algunos casos, consumo de sustancias psicoactivas, abuso sexual y violencia intrafamiliar y de pareja (Guevara Fletcher, 2011; Hérnandez Bello \& Gutiérrez, 2008; Ibáñez \& Moya, 2010; Mogollón-Pérez \& Vázquez, 2008; Mogollón Pérez \& Vázquez Navarrete, 2006).

Así mismo, los estudios que evaluaron la percepción del estado de salud de las personas en CDFI aseguraron que se presentaba una mayor percepción de enfermedad que en otras poblaciones vulnerables urbanas (Hérnandez Bello \& Gutiérrez, 2008; Mogollón Pérez \& Vázquez Navarrete, 2006).

Con relación al acceso al servicio de salud se encontró que las dificultades en el uso de este servicio se debían a: (a) barreras de tipo económico, siendo considerada la pobreza y la informalidad laboral las principales limitaciones para obtener el acceso; (b) barreras geográficas, por las amplias distancias que requerían una alta inversión de tiempo para movilizarse hasta el proveedor del servicio; (c) barreras administrativas, por el poco conocimiento sobre la tramitología, los horarios y lugares de atención; y (d) barreras culturales, puesto que se reportó que algunas personas en CDFI tenían poca confianza en la medicina convencional (Carrillo, 2010; Hérnandez Bello \& Gutiérrez, 2008; Mogollón-Pérez \& Vázquez, 2008; Prada Gómez et al., 2008; Sánchez Isaza et al., 2016).

Las condiciones de vivienda y asentamiento fueron investigadas por el $45.5 \%$ de los estudios. En general, se identificó alta presencia de hacinamiento y entornos de hábitat de riesgo. En algunos casos se afirmó que el estado de las viviendas de personas en CDFI era peor que el de la población receptora; aun cuando la segunda también se encontrara en situación de vulnerabilidad socioeconómica 
(Guevara Fletcher, 2011; Hérnandez Bello \& Gutiérrez, 2008; Mogollón-Pérez \& Vázquez, 2008).

El acceso a servicios públicos fue investigado por el $27.3 \%$ de los estudios. Los hallazgos señalaron que el acceso era limitado debido a que las viviendas se encontraban en zonas con déficit de cobertura empresarial y, en los casos en que se tenía acceso, gran parte de los ingresos del hogar se destinaban al pago de estos (Dueñas et al., 2014; Guevara Fletcher, 2011; Hérnandez Bello \& Gutiérrez, 2008; Mogollón-Pérez \& Vázquez, 2008; Mogollón Pérez \& Vázquez Navarrete, 2006).

La tenencia de propiedades y/o bienes fue investigada por el $\mathbf{2 6 . 9 \%}$ de los estudios. Las consecuencias más directas del DFI eran la pérdida de bienes por el agotamiento de activos; puesto que las personas eran obligadas a abandonar la tierra $y$, por ende, perder sus cultivos, herramientas y maquinaria y cuando llegaban a la ciudad la consecución de nuevo patrimonio se tornaba difícil (Arias et al., 2014; Carrillo, 2010; Mogollón-Pérez \& Vázquez, 2008; Patiño \& Herrán, 2013).

La situación laboral fue investigada por el $76.9 \%$ de los estudios. Se reportó que al llegar a la ciudad algunos hogares experimentaban una transformación en los roles de género. Puesto que en los contextos urbanos se ampliaba la oferta de trabajo para las mujeres, situación que las exponía con frecuencia a asumir la carga económica del hogar, además de los roles de cuidado. Sin embargo, esto no aseguraba que los trabajos fueran estables y bien remunerados. Dentro de las actividades laborales más reportadas se encontraban: el servicio doméstico, actividades de emprendimiento o venta ambulante en el caso de las mujeres; y en el caso de los hombres albañilería y venta ambulante (Aysa-Lastra, 2011; Carrillo, 2010; Castiblanco Moreno, 2016; Dueñas et al., 2014; Machado-Duque \& Calderón-Flórez, 2014; Pareja Amador \& Domínguez, 2014; Prada Gómez et al., 2008).

Los estudios también reportaron que las personas en CDFI competían en el mercado laboral con la desventaja de contar con escasos niveles educativos y sufrir el estigma de ser desplazados. Por lo que se enfrentaban a condiciones laborales precarias que reducían la calidad de vida y la cantidad de dinero destinado a la satisfacción de necesidades básicas (Aysa-Lastra, 2011; Carrillo, 2010; Castiblanco Moreno, 2016; Machado-Duque \& Calderón-Flórez, 2014; Prada Gómez et al., 2008).

El nivel de escolaridad y el acceso a la educación fue investigado por el $46.2 \%$ de los estudios. Se reportó un escaso nivel educativo, lo que representaba una desventaja frente a la población que habitaba en los lugares de asentamiento, especialmente, frente a las oportunidades y condiciones laborales (Aysa-Lastra, 2011; Herrán \& Patiño, 2015; Silva Arias \& Guataquí Roa, 2008; Silva Arias \& Sarmiento Espinel, 2013).

Con relación al acceso a la educación se identificó que el DFI implicó sacrificar temporalmente la asistencia a los planteles educativos por parte de los integrantes de la familia en edad escolar, lo que se relacionaba con menor nivel educativo comparado con el resto de la población del lugar de asentamiento. Factor que repercutía en un decremento importante de posibilidades para ingresar al mercado laboral formal (Aysa-Lastra, 2011; Bozzoli et al., 2012; Carrillo, 2010; Hérnandez Bello \& Gutiérrez, 2008; Muggah, 2000).

El acceso a ayuda humanitaria y programas sociales fue investigado por el $15.4 \%$ de los estudios. Al respecto, se reportó que las personas en CDFI no eran autosuficientes ni lograban satisfacer sus necesidades básicas sin la ayuda permanente de entidades de gobierno $y / u$ organizaciones de carácter humanitario. Sin embargo, los programas eran insuficientes para proporcionar estabilidad (Carrillo, 2010; Espinosa Menéndez et al., 2012).

\section{Análisis estadístico de datos textuales}

Se utilizó el paquete estadístico SPAD® (versión 9.0) para analizar y sintetizar el contenido de los estudios. Inicialmente se realizó la segmentación del corpus textual. En la tabla 2 se encuentra la información sobre la longitud y la riqueza del texto de los apartados de título, resumen, palabras clave, resultados y discusión de los estudios incluidos en esta revisión. El corpus tuvo una longitud de 36.285 palabras y estuvo conformado por 4.940 palabras distintas. Se aplicó un umbral de frecuencia 7 que permitió mantener el $75 \%$ del corpus. Además, se utilizó un análisis de contenido para reducir el vocabulario, se eliminaron las palabras vacías (artículos, preposiciones y conjunciones) y, de acuerdo con los objetivos de la presente investigación, se mantuvieron aquellas con sentido semántico.

Tabla 2. Corpus textual de trabajo

\begin{tabular}{ll}
\hline Análisis lexicométrico & \\
\hline Número total de palabras & 36.285 \\
Número total de palabras distintas & 4.940 \\
Porcentaje del corpus mantenido & $75 \%$ \\
\hline
\end{tabular}

Después, se efectuó un análisis factorial de correspondencias, se incluyeron como variable textual las 60 palabras de mayor frecuencia de aparición y peso en la construcción del factor 1 y 2 como se observa en la tabla 3 . Como variables categóricas se seleccionaron: el periodo de publicación, el lugar de publicación, la metodología, el tipo de muestra y el género con el fin de obtener una visión gráfica y simplificada de la información como se observa en la figura 2. En este análisis, el primer factor conserva el $26.87 \%$ y el segundo el $21.72 \%$ de la varianza. Por tanto, la representación del primer plano factorial conserva el $48.59 \%$ de la varianza total. Se señala la existencia de tipologías temáticas que aluden a nivel horizontal a periodo y lugar de publicación (factor 1), y a nivel vertical a metodología, tipo de muestra y género (factor 2).

En lo que se refiere a los cuadrantes, se puede observar que en el cuadrante superior derecho se ubicaron, principalmente, los estudios realizados con metodología cuantitativa que contaron con la participación de muestras de género femenino y masculino y trataron temáticas relacionadas con la tierra, los lugares de expulsión y recepción, los ingresos, la calidad y el bienestar, así como la generación de redes y oportunidades de las personas en CDFI.

En el cuadrante superior izquierdo se ubicaron los estudios publicados, principalmente, en América del Sur que trabajaron con familias y población infantil e investigaron temáticas centradas en la seguridad y la economía de los hogares, las condiciones de salud y, específicamente, de seguridad alimentaria y hallaron déficit nutricional y riesgo con énfasis en el grupo infantil. 
Tabla 3. Palabras con mayor frecuencia de aparición

\begin{tabular}{|c|c|c|c|c|c|}
\hline Palabra & Frecuencia & Palabra & Frecuencia & Palabra & Frecuencia \\
\hline Hogares & 127 & Recepción & 33 & Tierra & 20 \\
\hline Alimentos & 122 & Educación & 32 & Comida & 19 \\
\hline Salud & 104 & Menores & 32 & Estrategias & 19 \\
\hline Mujeres & 99 & Inseguridad & 31 & Varones & 19 \\
\hline Condiciones & 84 & Violencia & 28 & Impacto & 18 \\
\hline Migrantes & 82 & Económica & 27 & Adaptación & 17 \\
\hline Consumo & 65 & Municipios & 27 & Calidad & 17 \\
\hline Trabajo & 65 & Necesidades & 27 & Ciudades & 17 \\
\hline Atención & 59 & Pobreza & 26 & Derechos & 17 \\
\hline Ingresos & 59 & Programas & 26 & Pérdida & 17 \\
\hline Seguridad & 53 & Bienestar & 24 & Armado & 16 \\
\hline Laboral & 51 & Cambios & 24 & Desnutrición & 16 \\
\hline Servicios & 48 & Compra & 24 & Oportunidades & 16 \\
\hline Conflicto & 44 & Victimas & 24 & Redes & 16 \\
\hline Niños & 42 & Comunidades & 23 & Política & 15 \\
\hline Social & 41 & Nutricional & 22 & Rurales & 15 \\
\hline Empleo & 39 & Expulsión & 21 & Autoempleo & 14 \\
\hline Familia & 38 & Vulnerabilidad & 21 & Sostenibilidad & 14 \\
\hline Riesgo & 38 & Gasto & 20 & Trabajadores & 14 \\
\hline Mercado & 36 & Hijos & 20 & Déficit & 13 \\
\hline
\end{tabular}

En el cuadrante inferior izquierdo, se ubicaron los estudios realizados con metodologías cualitativa y mixta que contaron con muestras de personas adultas, principalmente, de género femenino. Estos estudios trataron temáticas centradas en las pérdidas, los cambios y los procesos de adaptación, así como las labores de crianza de las mujeres y las necesidades a nivel social y de salud.

Por último, en el cuadrante inferior derecho se situaron los estudios publicados en su mayoría en América Central y Europa que trabajaron con datos oficiales y evaluaron las condiciones de vulnerabilidad y pobreza de las personas en CDFI. Estos estudios hicieron énfasis en la situación laboral y de generación de ingresos que se ve comprometida por desventajas en la formación educativa, lo que lleva incluso a la generación de alternativas como el autoempleo.

\section{Discusión}

En Colombia el DFI es el hecho que más ha afectado a la población en el marco del conflicto armado y se convierte en uno de los mayores desafíos para el Estado. El DFI representa una de las crisis humanitarias más relevantes de los últimos tiempos debido a las profundas secuelas que genera a nivel individual, social y económico. En medio de la inestabilidad que supone la migración forzada, una de las necesidades más apremiantes que enfrentan las personas afectadas es reestructurar su área económica. Puesto que se dificulta la consecución de fuentes permanentes de ingreso y la satisfacción de necesidades. Por tanto, esta revisión sistemática de la literatura científica realizó una síntesis acerca de los hallazgos más recientes en investigación sobre prácticas de consumo y fuentes de ingreso de las personas en CDFI en Colombia.

Los resultados sugieren que el DFI tiene profundas repercusiones negativas en la satisfacción de necesidades básicas de alimentación y acceso a servicios. Además de que genera alteraciones en las condiciones de salud física y psicológica. Realidad producto, principalmente, de la dependencia financiera, las barreras geográficas, culturales y sociales así como las transformaciones en la estructura familiar que experimentan las personas en CDFI (Gamboa-Delgado et al., 2007; Guevara Fletcher, 2011; Hayes-Conroy \& Sweet, 2014; Hérnandez Bello \& Gutiérrez, 2008; Herrán \& Patiño, 2015; Machado-Duque \& Calderón-Flórez, 2014; Prada et al., 2010; Prada Gómez et al., 2008).

Algunos de los estudios son concluyentes en plantear que el bajo nivel educativo, el cambio del contexto rural al urbano y la competencia en condiciones de desigualdad con la población residente en los lugares de asentamiento son factores que influyen en las pocas oportunidades de acceder al sector laboral formal por parte de las personas en 


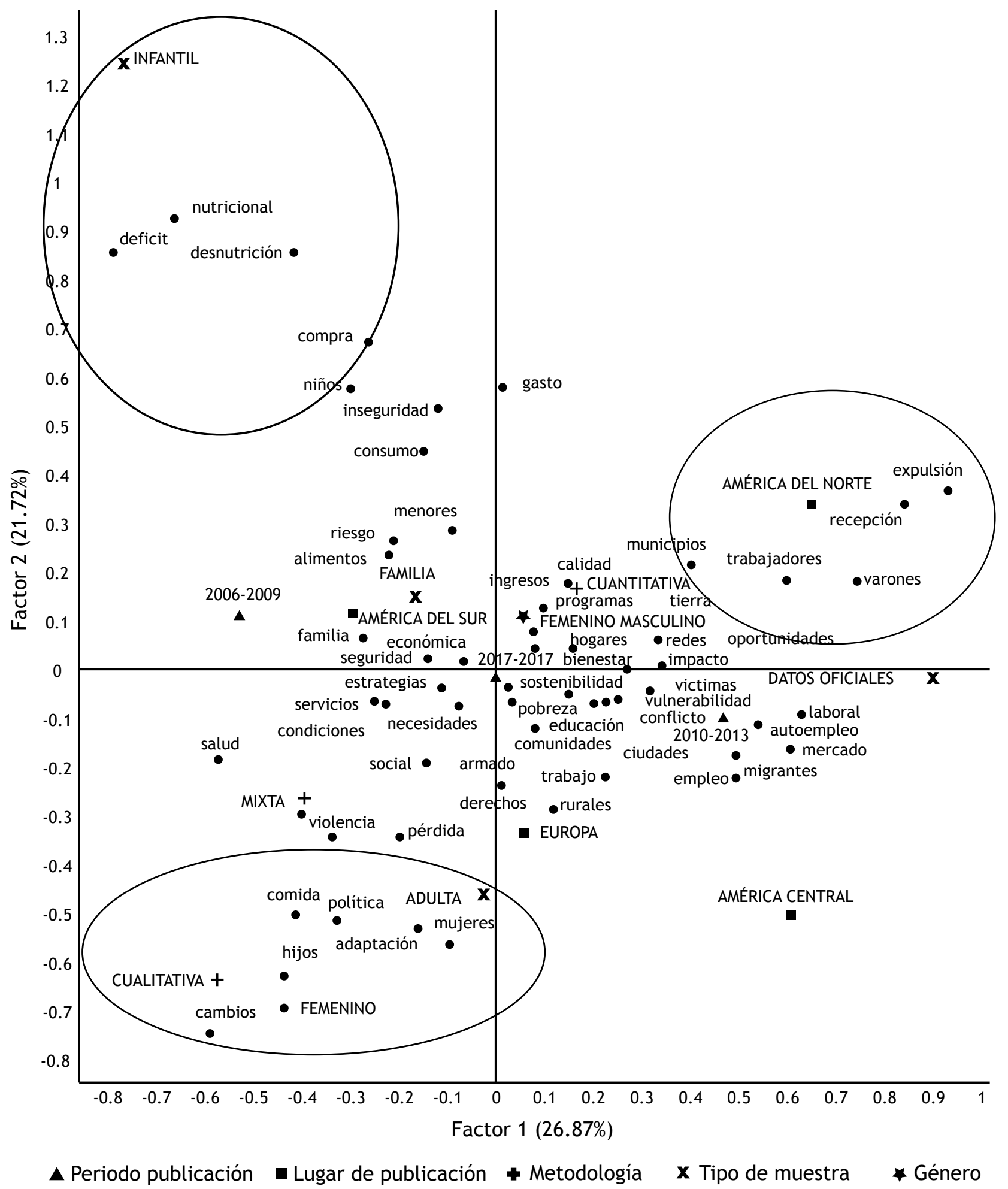

Figura 1. Plano factorial de palabras con sentido semántico más contributivas para los dos factores de los estudios analizados sobre las prácticas de consumo y fuentes de ingreso de las personas en CDFI en Colombia

CDFI (Aysa-Lastra, 2011; Bozzoli et al., 2012; Carrillo, 2010; Dueñas et al., 2014; Hérnandez Bello \& Gutiérrez, 2008; Machado-Duque \& Calderón-Flórez, 2014; Prada Gómez et al., 2008)

Además, todos los estudios coinciden en afirmar que el DFI representa una pérdida material, social, económica y de salud que deja una huella profunda en la vida de las personas que son víctimas directas. Por lo que se puede afirmar que la población en CDFI constituye uno de los grupos de mayor vulnerabilidad a nivel nacional.

Por tanto, el DFI en Colombia es una de las crisis humanitarias más relevantes en los últimos tiempos y es un 
fenómeno que atenta contra la calidad de vida de quienes además de ser víctimas directas del conflicto armado se enfrentan a situaciones de exclusión y/o vulnerabilidad en el lugar de asentamiento.

\section{Limitaciones}

Esta revisión sistemática tiene una serie de limitaciones $y$, por lo tanto, debe interpretarse con cautela. A pesar de nuestra estrategia de búsqueda sistemática, es probable que ciertos estudios no publicados, no indexados o que emplearon otros términos para el fenómeno de interés no hayan sido incluidos. También, es necesario señalar que no establecimos contacto directo con autores.

Se debe tener precaución con generalizar los resultados presentados a toda la población en CDFI en Colombia. Esto debido a cuestiones de carácter metodológico y empírico.

A nivel metodológico, como se señaló en los resultados descriptivos fueron escasos los estudios con diseño longitudinal. Si bien esto se puede deber a la complejidad de lograr un contacto permanente con población en CDFI, es claro que los diseños transversales tienen limitaciones para establecer relaciones causales o conocer la evolución en las condiciones socioeconómicas de este grupo poblacional. También, es necesario también trabajar en relación con la estandarización de instrumentos de medición. Si bien se investigan temas diversos y se emplean múltiples fuentes, es importante fortalecer aspectos psicométricos de los instrumentos de medición. De hecho, pocos estudios presentan información sobre confiabilidad o validez. Tampoco fue viable realizar un metaanálisis, por lo tanto, la variabilidad entre los estudios y el sesgo de publicación no se examinaron.

A nivel empírico, esta revisión no identifico artículos que hicieran comparaciones entre grupos en función del tiempo que llevaran las personas en CDFI o el tipo de desplazamiento (individual o masivo). Entendemos que estos no son aspectos fáciles de abordar. Sin embargo, es claro que el tiempo y el tipo de DFI puede ser un fuerte predictor sobre la estabilidad socioeconómica. Tampoco se encontraron estudios que investigaran el DFI desde una perspectiva de consumo y es evidente que la población en CDFI es consumidora de bienes, servicios y política pública. Por lo que se hace necesario continuar con el estudio de esta problemática desde una perspectiva de consumo que permita obtener hallazgos que sean empleados para la transformación y promoción de políticas públicas que tengan como objetivo generar bienestar en las personas en CDFI en Colombia.

Además, se identificó que son escasos los estudios que exploraron sobre el acceso a ayuda humanitaria. Sin embargo, es evidente que el DFI enfrenta, muchas veces, a las personas obligadas a migrar a que su principal fuente de subsistencia sea la ayuda permanente de entidades del gobierno y/u organizaciones de carácter humanitario. Por último, esta revisión no identificó ningún estudio que indagara sobre las prácticas de ocio y recreación, sin embargo, este es un aspecto trascendental en relación con el bienestar general. Por tanto, recomendamos que la futura investigación aporte evidencia empírica al respecto.

\section{Implicaciones prácticas y conclusión}

Colombia se enfrenta al desafío de construir una paz estable y duradera. Sin embargo, la violencia sociopolítica a la que ha estado expuesta la sociedad ha dejado profundas huellas y aún más en aquellas personas víctimas directas del conflicto armado.

Puntualmente, en relación con el DFI, que es el hecho que más víctimas ha cobrado y que ubica a Colombia dentro de los países con más población en CDFI en el mundo, el Estado ha propuesto políticas públicas para reparar a las víctimas y prevenir nuevos DFI. Algunas de estas medidas son la Ley 387 (Congreso de la República de Colombia, 1997) y la Ley 1448 (Congreso de la República de Colombia, 2011). Sin embargo, es evidente que estas medidas resultan aún insuficientes respecto a las necesidades que enfrentan las personas en CDFI.

Por tanto, a nivel de intervención, partiendo de los resultados obtenidos en esta revisión, se recomienda incentivar programas, tanto a corto como a mediano plazo, que trabajen en mitigar barreras culturales, sociales, educativas y económicas.

En resumen, esta revisión sistemática indica que: (1) en términos de alimentación, que es una de las necesidades más apremiantes de esta población, se debe propender por proporcionar una dieta que asegure los requerimientos mínimos nutricionales; (2) en términos de salud, se deben buscar soluciones innovadoras que disminuyan los impedimentos de acceso y/o cobertura (algunas acciones prácticas como la telemedicina podrían apoyar al respecto); (3) en términos educativos, factor identificado como impedimento en personas en CDFI para acceder a empleo formal, se deben generar programas que reduzcan las diferencias entre población receptora y personas en CDFI; (4) en términos de fuentes de ingreso, se deben generar programas de formación práctica que favorezcan las posibilidades de acceder al sector laboral formal. Pero, sobre todo, resulta importante señalar que se debe propender por generar programas de intervención de largo plazo que permitan un restablecimiento de derechos y condiciones de vida óptimas para las personas en CDFI. Puesto que es la única manera de generar escenarios de reconciliación, impactar directamente en la construcción de paz y promover crecimiento nacional.

Sumado a esto, resulta importante enfatizar que las personas en CDFI, muchas veces, se enfrentan a circunstancias de humillación o exclusión en los lugares de asentamiento. Por lo que es necesario señalar que desde el proceder social y ético esta debe ser una prioridad en el momento de generar intervenciones.

Por último, queremos señalar que es importante continuar avanzando en esta línea de investigación con el fin de dar cuenta de las necesidades y limitaciones a las que se enfrenta la población en CDFI y con base en la evidencia científica alertar a las entidades estatales sobre las principales necesidades y si es el caso plantear reformulaciones en la política pública.

\section{Agradecimientos}

La investigación presentada en este artículo fue financiada por la Fundación Universitaria Konrad Lorenz de Colombia y por la convocatoria 645-2014 de Jóvenes Investigadores e Innovadores de Colciencias.

\section{Contribuciones de autor}

Las dos autoras contribuyeron a todas las etapas de la revisión. Igualmente, aprobaron la versión final del manuscrito. 


\section{Referencias}

Arias, M. A., Ibáñez, A. M., \& Querubín, P. (2014). The desire to return during civil war: Evidence for internally displaced populations in Colombia. Peace Economics, Peace Science and Public Policy, 20(1), 209-233. http://dx.doi.org/10.1515/peps-2013-0054

Aysa-Lastra, M. (2011). Integration of internally displaced persons in urban labour markets: A case study of the IDP population in Soacha, Colombia. Journal of Refugee Studies, 24(2), 277-303. http://dx.doi.org/10.1093/jrs/feq054

Bohada, M. (2010). Desplazamiento forzado y condiciones de vida de las comunidades de destino: el caso de Pasto, Nariño. Revista de Economía Institucional, 12(23), 259-298.

Bozzoli, C., Bruck, T., \& Wald, N. (2012). Self-employment and Conflict in Colombia. Journal of Conflict Resolution, 57(1), 117142. http://dx.doi.org/10.1177/0022002712464849

Carranza Romero, J. E., Dueñas Herrera, X., \& González Espitia, C. G. (2011). Análisis empírico de la relación entre la actividad económica y la violencia homicida en colombia. Estudios Gerenciales, 27(119), 59-77. http://dx.doi.org/10.1016/S0123-5923 (11)70157-7

Carrillo, A. C. (2010). Internal displacement in Colombia: Humanitarian, economic and social consequences in urban settings and current challenges. International Review of the Red Cross, 91(875), 527. http://dx.doi.org/10.1017/S1816383109990427

Castiblanco Moreno, S. E. (2016). Female entrepreneurship in a forced displacement situation: The case of Usme in Bogota. Suma de Negocios, 7(15), 61-72. http://dx.doi.org/10.1016/J.SUMNEG.2016. 02.004

Congreso de la República de Colombia. (1997). Ley 387. Por la cual se adoptan medidas para la prevención del desplazamiento forzado; la atención protección, consolidación y estabilización socioeconómica de los desplazados internos por la violencia en la República de Colombia.

Congreso de la República de Colombia. (2011). Ley 1448. Por la cual se dictan medidas de atención, asistencia y reparación integral a las víctimas del conflicto armado interno y se dictan otras disposiciones, Pub. L. No. Ley 1448. Colombia. Recuperado de https://www.unidadvictimas.gov.co/es/ley-1448-de-2011/13653

Dueñas, X., Palacios, P., \& Zuluaga, B. (2014). Forced Displacement in Colombia: What determines expulsion and reception in municipalities? Peace Economics, Peace Science and Public Policy, 20(4), 585-597. http://dx.doi.org/10.1515/peps-2014-0037

Espinosa Menéndez, N., Galindo Rosero, A., Bastidas Lopera, W., \& Monsalve Rojas, J. E. (2012). Estado actual de la población víctima de desplazamiento forzado en cuanto al enfoque diferencial de su atención, el derecho a la integridad, la subsistencia mínima y al ingreso económico. El Ágora USB, 12(1), 19-46.

Gamboa-Delgado, E. M., López-Barbosa, N., Vera-Cala, L. M., \& Prada-Gómez, G. E. (2007). Patrón alimentario y estado nutricional en niños desplazados en Piedecuesta, Colombia. Revista de Salud Pública, 9(1), 129-139.

Guevara Fletcher, D. A. (2011). Discursos y disciplinamiento de la pobreza en la población desplazada por la violencia en Colombia. OBETS: Revista de Ciencias Sociales. Instituto Interuniversitario de Desarrollo Social y Paz. http://dx.doi.org/10.14198/ OBETS2011.6.2.04

Hayes-Conroy, A., \& Sweet, E. L. (2014). Whose adequacy? (Re) imagining food security with displaced women in Medellín, Colombia. Agriculture and Human Values. http://dx.doi.org/10. 1007/s10460-014-9546-y

Hérnandez Bello, A., \& Gutiérrez. (2008). Vulnerabilidad y exclusión: Condiciones de vida, situación de salud y acceso a servicios de salud de la población desplazada por la violencia asentada en Bogotá-Colombia, 2005. Revista Gerencia y Políticas de Salud, 7(14), 145-176.
Herrán, O., \& Patiño, G. (2015). Sostenibilidad alimentaria en hogares con condición de desplazamiento forzado en el área metropolitana de Bucaramanga, Colombia. Revista de la Facultad de Medicina, 62(1Sup), 19-27. http://dx.doi.org/10.15446/revfacmed.v62n3sup.40739

Ibáñez, A. M., \& Moya, A. (2010). Vulnerability of victims of civil conflicts: Empirical evidence for the displaced population in Colombia. World Development, 38(4), 647-663. http://dx.doi.org/10.1016/j. worlddev.2009.11.015

Ibáñez, A. M., \& Vélez, C. E. (2008). Civil conflict and forced migration: The micro determinants and welfare losses of displacement in Colombia. World Development, 36(4), 659-676. http:// dx.doi.org/10.1016/j.worlddev.2009.11.015

Machado-Duque, M. E., \& Calderón-Flórez, V. (2014). Determinantes socioeconómicos, inseguridad alimentaria y desnutrición crónica en población desplazada de primera infancia, Pereira, Colombia. Revista Médica de Risaralda, 20(1), 3-8.

Macuacé, R. (2009). Una aproximación socioeconómica a los impactos del fenómeno del desplazamiento forzado en los municipios de el Tambo y Timbío. Biotecnología en el Sector Agropecuario y Agroindustrial, 7(1), 91-101.

Mogollón-Pérez, A. S., \& Vázquez, M. L. (2008). Factores que inciden en el acceso de la población desplazada a las instituciones prestadoras de servicios de salud en Colombia. Cadernos de Saúde Pública, 24(4), 745-754. http://dx.doi.org/10.1016/j. worlddev.2009.11.015

Mogollón Pérez, A. S., \& Vázquez Navarrete, M. L. (2006). Opinión de las mujeres desplazadas sobre la repercusión en su salud del desplazamiento forzado. Gaceta Sanitaria, 20(4), 260-265.

Montero, I., \& León, O. G. (2007). A guide for naming research studies in psychology. International Journal of Clinical and Health Psychology, 7(3), 847-862.

Moreno, M. C. (2005). La investigación cualitativa en marketing: el camino hacia una percepción social del mercado. Revista Colombiana de Marketing, 4(6), 68-85.

Muggah, R. (2000). Through the developmentalist's looking glass: Conflict-induced displacement and involuntary resettlement in Colombia. Journal of Refugee Studies, 13(2), 133-164.

Pareja Amador, A. J., \& Domínguez, A. I. (2014). Violencia contra la mujer y desplazamiento forzado. Análisis de las estrategias de vida de jefas de hogar en Medellín. Acta Sociológica, 2014(65), 151-171. http://dx.doi.org/10.1016/S0186-6028(14)70240-3

Patiño, G. A., \& Herrán, O. F. (2013). Desplazamiento forzado, niñez y adolescencia: Escenarios en relación con su estabilización socioeconómica. Revista de Salud Pública, 14(2 suppl.), 58-68.

Petticrew, M., \& Roberts, H. (2008). Systematic reviews in the social sciences: A practical guide.

Prada, G. E., Herrán, O. F., \& Villamizar, I. (2010). Caracterización del consumo de alimentos en familias desplazadas del municipio de Lebrija, Santander. Colombia. 2006. Revista de La Universidad Industrial de Santander. Salud, 42(1), 18-24.

Prada Gómez, G. E., Herrán Falla, O. F., \& Ortiz Cárdenas, R. (2008). Patrón alimentario y acceso a los alimentos en familias desplazadas en el municipio de Girón, Santander, Colombia. Revista Panamericana de Salud Pública, 23(4), 257-263. http://dx.doi. org/10.1590/S1020-49892008000400005

Sánchez Isaza, D. C., Cataño Ordóñez, N., \& Ordóñez, N. C. (2016). Factores de riesgo total en familias migrantes en Soledad, Atlántico. Revista Cuidarte, 7(1), 1152. http://dx.doi.org/10.15649/ cuidarte.v7i1.174

Silva, A. C., \& Guataquí, J. C. (2011). ¿Selección positiva o negativa? Inserción de la migración interna y el desplazamiento forzado en el mercado laboral urbano de Colombia 2001-2006. Estudios Demográficos y Urbanos, 26(1), 113-140. 
Silva Arias, A. C., \& Guataquí Roa, J. C. (2008). Caracterización instrumental del desplazamiento forzado en Colombia: hechos estilizados y la Encuesta Continua de Hogares (2001-2006). Revista de Ciencias Sociales, 14(3), 439-452.

Silva Arias, A. C., \& Sarmiento Espinel, J. A. (2013). Desplazados forzados y su participación en el mercado laboral colombiano. Revista Facultad de Ciencias Económicas: Investigación y Reflexión, 21(1), 167-187.
Urrutia, G., \& Bonfill, X. (2010). [Prisma declaration: a proposal to improve the publication of systematic reviews and meta-analyses]. Medicina Clínica, 135(11), 507-511. http://dx.doi.org/10. 1016/j.medcli.2010.01.015 